\title{
Relación entre la composición, la capacidad antioxidante y el contenido fenólico de alimentos concentrados para perros
}

\author{
Relationship between composition, antioxidant capacity and phenolic content of \\ feed concentrate for dogs \\ Giovanni Restrepo B. ${ }^{1,5}$, Alexandra Usuga S. ${ }^{2}$, Luis Miguel Gomez ${ }^{3}$, Carolina Mesa \\ Pineda $^{3}$, Oliver Restrepo Rojas ${ }^{3}$, Juan Camilo Duque ${ }^{3}$, Benjamín A. Rojano ${ }^{4}$
}

\section{Resumen}

\begin{abstract}
El estrés oxidativo se ha asociado con el desarrollo de muchas enfermedades crónicas y degenerativas en los caninos. La presencia de antioxidantes en los alimentos contribuye a la reducción de los efectos nocivos de las especies reactivas de oxígeno (EROS) y nitrógeno (ERNS). Los polifenoles son un grupo de sustancias con alta actividad antioxidante y juegan un papel importante en la prevención de enfermedades. El objetivo de esta investigación fue evaluar la relación entre la composición, la capacidad antioxidante y el contenido de polifenoles de concentrados para perros de diferentes segmentos comerciales. Se seleccionaron aleatoriamente 19 alimentos para perros de cuatro segmentos comerciales: popular, económico, premium y super premium. Mediante un análisis bromatológico se evaluó la composición de cada alimento. Se midió la capacidad antioxidante total (CAT) y el contenido fenólico total (FT) a través de los métodos ABTS y Folin-Ciocalteu, respectivamente. Se realizaron análisis de varianza y de correlación. Los concentrados de los segmentos premium y super premium presentaron proporciones mayores de proteína cruda y grasa, y menores de fibra cruda, cenizas, calcio y fósforo en comparación con los concentrados de los segmentos popular y económico $(\mathrm{p}<0.05)$. Para los segmentos popular, económico, premium y super premium se encontraron valores de FT de $160.1 \pm 49.8,146.7 \pm 41.5,131.6 \pm 21.5$ y $144.9 \pm 34.1 \mathrm{mg}$ de ácido gálico/100 g de muestra y valores de CAT de $3146.0 \pm 564.5,2895.4 \pm 581.0,2837.6 \pm 241.7$ y $2875.79 \pm 325.8 \mu \mathrm{mol}$ Trolox/1 de muestra, respectivamente $(\mathrm{p}>0.05)$. Se encontró una
\end{abstract}

\footnotetext{
${ }^{1}$ Facultad de Ciencias Agrarias, Universidad Nacional de Colombia sede Medellín, Antioquia, Colombia

${ }^{2}$ Facultad de Medicina Veterinaria, Universidad CES, Medellín, Antioquia, Colombia

${ }^{3}$ Grupo de Investigación Nutri-Solla, Solla S.A, Itagüi, Antioquia, Colombia

${ }^{4}$ Facultad de Ciencias, Universidad Nacional de Colombia sede Medellín, Antioquia, Colombia

${ }^{5}$ E-mail: grestre0@unal.edu.co
}

Recibido: 28 de enero de 2019

Aceptado para publicación: 30 de agosto de 2019 
correlación positiva entre el contenido de fibra cruda y el FT $(r=0.47, p<0.05)$. Se concluye que la segmentación comercial de los concentrados para perros no está reflejada en su aporte antioxidante y que un mayor contenido de fibra cruda, propio de concentrados de los segmentos popular y económico, está asociado positivamente con el contenido de fenoles.

Palabras clave: alimento; antioxidantes; caninos; estrés oxidativo; fenoles

\title{
Abstract
}

\begin{abstract}
Oxidative stress has been associated with the development of many chronic and degenerative diseases in canines. The presence of antioxidants in food contributes to the reduction of the harmful effects of reactive oxygen species (ROS) and nitrogen (RNS). Polyphenols are a group of substances with high antioxidant activity and play an important role in the prevention of diseases. The aim of this study was to evaluate the relationship between the composition, the antioxidant capacity and the polyphenol content of dog feeds from different commercial segments. Nineteen dog foods from four commercial segments: popular, economic, premium and super premium were randomly selected. Through a bromatological analysis the composition of each feed was evaluated. Total antioxidant capacity (TAC) and total phenolic content (TFC) were measured through the ABTS and Folin-Ciocalteu methods, respectively. Analysis of variance and correlation were performed. Dog feeds of the premium and super premium segments presented higher proportions of crude protein and fat, and lower crude fiber, ash, calcium and phosphorus, in comparison with feeds of the popular and economic segments $(\mathrm{p}<0.05)$. For the popular, economic, premium and super premium segments, TFC values of $160.1 \pm 49.8,146.7 \pm$ $41.5,131.6 \pm 21.5$ and $144.9 \pm 34.1 \mathrm{mg}$ of gallic acid/100 $\mathrm{g}$ of sample and CAT values of $3146.0 \pm 564.5,2895.4 \pm 581.0,2837.6 \pm 241.7$ and $2875.79 \pm 325.8 \mu \mathrm{mol}$ Trolox $/ \mathrm{L}$ sample were determined, respectively ( $p>0.05$ ). A positive correlation was found between crude fiber content and TFC $(r=0.47, p<0.05)$. It is concluded that commercial segmentation of the dog feeds is not reflected in its antioxidant contribution and a higher crude fiber content, typical of feeds of the popular and economic segments, is positively associated with the content of phenols.
\end{abstract}

Key words: feed; antioxidants; canine; oxidative stress; phenols

\section{INTRODUCCIÓN}

Los antioxidantes se usan generalmente para ralentizar o detener la peroxidación de los lípidos y, en consecuencia, para preservar la frescura de productos que, como los alimentos para animales, pueden sufrir oxidación (Blaszczyk et al., 2013). Existen dos tipos de antioxidantes, naturales y sintéticos (Hilton, 1989). Dentro de los antioxidantes naturales se encuentran los tocoferoles, el ácido ascórbico y el extracto de romero como los más utilizados en alimentos, mientras que entre los antioxidantes sintéticos se encuentran el butilhidroxitolueno (BHT), el butilhidroxianisol (BHA) y el etoxiquin (EQ).

El estrés oxidativo se refiere a la lesión celular y al cambio patológico que ocurre cuando hay un desequilibrio que favorece a los oxidantes sobre los antioxidantes dentro de un organismo vivo (Soffler, 2007). Se han demostrado efectos benéficos en la salud de los perros por el suministro de antioxidantes en la dieta, a través de la reducción del estrés oxidativo (Baskin et al., 2000; Milgram et al., 2002; Pan et al., 2018). Incluso se ha eva- 
luado el efecto de otros suplementos dietarios en el estatus oxidativo de los caninos (Pacheco et al., 2018). Adicionalmente, se ha descrito la relación entre el estrés oxidativo de los perros y diferentes condiciones ambientales como restricciones sociales y espaciales, prácticas de manejo, condiciones de salud y diversas patologías (Macotpet et al., 2013; Passantino et al., 2014; Marquis et al., 2015).

Los niveles de antioxidantes individuales en los alimentos no reflejan necesariamente su capacidad antioxidante total (CAT), que depende de las interacciones sinérgicas y rédox entre las diferentes moléculas presentes en el alimento, de manera que, se han desarrollado varios métodos para medir la CAT de los alimentos (Pellegrini et al., 2003). Se conoce que la capacidad antioxidante puede ser influenciada por el contenido fenólico total del alimento (Prior et al., 1998). Los compuestos polifenólicos son un grupo cercano a 8000 sustancias que pueden ser clasificadas de acuerdo con su estructura. Los polifenoles en las frutas y hortalizas muestran una actividad antioxidante mayor que las vitaminas $\mathrm{C}$ y $\mathrm{E}$, y desempeñan un papel importante en la prevención de enfermedades crónicas (Zapata et al., 2014).

En la alimentación humana, la CAT se ha convertido en un aspecto prominente de la calidad y la funcionalidad de los alimentos y se utiliza con frecuencia para promover el consumo de tipos de alimentos con alta capacidad antioxidante (Pompella et al., 2014). Además, se sabe que la ingesta de antioxidantes de varias dietas y suplementos contribuye al estado de CAT de la población (Yang et al., 2011). Sin embargo, en la alimentación de mascotas, la CAT es un aspecto poco reportado y, posiblemente, poco considerado en la formulación de las dietas. Recientemente se encontró que los perros alimentados con sorgo tenían el mayor valor de capacidad de absorción de radicales de oxígeno (ORAC) en plasma sanguíneo (Corsato y Aldrich, 2018). El objetivo de esta investigación fue evaluar la relación entre la composición, la capacidad antioxidante y el contenido de polifenoles de alimentos concentrados para perros catalogados en varios segmentos comerciales.

\section{Materiales y Métodos}

\section{Alimentos Concentrados}

Se seleccionaron aleatoriamente 19 alimentos concentrados para perros de los cuatro segmentos comerciales existentes en Colombia. Cuatro de los concentrados fueron del segmento popular y cinco de cada uno de los segmentos económico, premium y super premium. Para la evaluación de la CAT y del contenido fenólico total (FT) se seleccionó una porción representativa de cada empaque comercial y se determinó la participación de cada tipo de pellet en la porción para su inclusión proporcional en el estudio.

\section{Análisis Bromatológico}

Se realizó la estimación de los valores bromatológicos para cada alimento concentrado de acuerdo con las siguientes metodologías: humedad (NTC 4888), proteína cruda (método Kjeldahl), grasa cruda (AOAC 13032; NTC 4969), cenizas (NTC 4648), fibra cruda (AOAC 978.10, NTC 5122), calcio (ICONTEC, 1998; NTC 302) y fósforo (ICONTEC, 2001; NTC 4981) (ICONTEC, 2019; AOAC, 2019).

\section{Capacidad Antioxidante Total (CAT)}

Se evaluó por triplicado la CAT de cada alimento concentrado mediante la prueba ABTS (Arts et al., 2004). Se maceraron $0.8 \mathrm{~g}$ de concentrado y se adicionaron $10 \mathrm{ml}$ de agua tipo I. Las preparaciones se sometieron a un vórtex por $30 \mathrm{~s}$ y luego se centrifugaron por $10 \mathrm{~min}$ a $5000 \mathrm{rpm}$ en una centrífuga $Z$ 206 A (Hermle, Alemania). Se utilizaron $10 \mu 1$ del sobrenadante y $990 \mu 1$ de la solución del radical $\mathrm{ABTS}^{+}$(Sigma-Aldrich, USA). Luego de 30 min de reacción a temperatura am- 
biente y en la oscuridad, se midió en un espectrofotómetro $6405 \mathrm{UV} / \mathrm{Vis}$ (Jenway, USA) el cambio en la absorbancia respecto a una solución referencia compuesta por 10 il de solución buffer y $990 \mu 1$ de la solución del radical $\mathrm{ABTS}^{+}$. El radical se generó por oxidación de $3.5 \mathrm{mM}$ de ABTS con $1.25 \mathrm{mM}$ de persulfato de potasio. Después de $24 \mathrm{~h}$ de reacción, se ajustó la absorbancia con PBS a pH 7.4 hasta 0.70 unidades, a una $\lambda$ de 732 nm $y$ se comparó contra una curva patrón con Trolox ${ }^{\circledR}$ (Sigma-Aldrich, USA).

\section{Contenido Fenólico Total (FT)}

El FT se evaluó a través del método de Folin-Ciocalteu, según lo descrito por Zapata et al. (2013). Se elaboró una curva patrón empleando como estándar el ácido gálico. El contenido de fenoles totales se expresó como $\mathrm{mg}$ de ácido gálico/100 g de muestra. Se empleó un espectrofotómetro UV-VIS (Jenway, 6405, Essex, Inglaterra). Las lecturas se realizaron por triplicado a $760 \mathrm{~nm}$. El reactivo Follin-Ciocalteu y el ácido gálico se obtuvieron de la casa comercial Merck (Alemania).

\section{Análisis Estadístico}

Se realizó un análisis de varianza, se validó la normalidad de los datos a través de la prueba Kolmogórov-Smirnov. La comparación de las medias para FT y CAT entre los diferentes segmentos comerciales de concentrados para perros se realizó mediante la prueba de Tukey. Todos los análisis se desarrollaron mediante el software SAS 9.2.

\section{Resultados y Discusión}

La industria de las mascotas es un sector importante en los Estados Unidos, con un gasto total para el año 2016 de US\$ $66.75 \mathrm{mil}$ millones, de los cuales US\$28.23 mil millones corresponde a gastos en alimento (Glodde et al., 2018). Los concentrados para mascotas son comúnmente clasificados en diferentes segmentos comerciales, entre los cuales se presentan variaciones en la composición y las materias primas utilizadas. Por ejemplo, la proporción de lípidos en los alimentos para mascotas puede variar entre $5 \mathrm{y}$ $40 \%$ de la dieta, donde la fuente de grasa puede ser animal, vegetal o, en ocasiones, una mezcla de ambos (Bauer, 2007). En el presente estudio, los concentrados de los segmentos premium y super premium presentaron una mayor proporción de proteína cruda, los concentrados super premium tuvieron un mayor contenido de grasa, mientras que los concentrados de los segmentos popular y económico tuvieron un mayor contenido de fibra cruda $(\mathrm{p}<0.05)$ (Cuadro 1).

Uno de los retos de las empresas productoras de concentrados está en la conservación de estos alimentos en condiciones de mínimo deterioro. La oxidación de los lípidos, especialmente de los ácidos grasos poliinsaturados afecta adversamente el sabor, la textura, el color, el olor y el valor nutricional de los alimentos durante el almacenamiento (Glodde et al., 2018). Se ha reportado que la oxidación de lípidos en la dieta en perros en crecimiento retrasa su desarro1lo, deteriora su estado antioxidante y debilita algunas de sus funciones inmunitarias (Turek et al., 2003). Por lo anterior, comúnmente se usan los aditivos alimentarios, los cuales son sustancias naturales o sintéticas que se agregan intencionalmente a los alimentos. En pequeñas cantidades, estas sustancias aumentan la durabilidad del producto alimenticio y mejoran o modifican sus propiedades, incluyendo su apariencia, sabor o estructura, sin disminuir el valor nutricional. De acuerdo con las características o composición del alimento, se necesita usar algunos de estos aditivos con propiedades antioxidantes de manera simultánea, lo cual genera una acción sinérgica (Silva y Lidon, 2016).

Muchos antioxidantes naturales, como los tocoferoles, la vitamina $\mathrm{Cy}$ los flavonoides, pueden ser efectivos en la conservación de alimentos por un corto periodo de tiempo; por lo tanto, antioxidantes sintéticos como el BHT, 
Cuadro 1. Composición de alimentos concentrados para perros según el segmento comercial

\begin{tabular}{lccccccc}
\hline Segmento & Humedad & $\begin{array}{c}\text { Proteína } \\
\text { cruda }\end{array}$ & Grasa & $\begin{array}{c}\text { Fibra } \\
\text { cruda }\end{array}$ & Cenizas & Calcio & Fósforc \\
\hline Popular & $7.73 \pm$ & $19.45 \pm$ & $10.48 \pm$ & $3.60 \pm$ & $7.32 \pm$ & $1.65 \pm$ & $1.11 \pm$ \\
& $1.13^{\mathrm{a}}$ & $0.58^{\mathrm{a}}$ & $1.29^{\mathrm{b}}$ & $0.59^{\mathrm{a}}$ & $0.78^{\mathrm{a}}$ & $0.35^{\mathrm{a}}$ & $0.14^{\mathrm{a}}$ \\
Económico & $7.78 \pm$ & $19.97 \pm$ & $10.62 \pm$ & $3.43 \pm$ & $7.74 \pm$ & $1.61 \pm$ & $1.12 \pm$ \\
& $0.23^{\mathrm{a}}$ & $2.45^{\mathrm{a}}$ & $2.00^{\mathrm{b}}$ & $0.84^{\mathrm{a}}$ & $0.94^{\mathrm{a}}$ & $0.22^{\mathrm{a}}$ & $0.17^{\mathrm{a}}$ \\
Premium & $8.03 \pm$ & $23.56 \pm$ & $11.42 \pm$ & $2.20 \pm$ & $6.85 \pm$ & $1.47 \pm$ & $0.94 \pm$ \\
& $0.77^{\mathrm{a}}$ & $1.21^{\mathrm{b}}$ & $1.70^{\mathrm{ab}}$ & $0.63^{\mathrm{b}}$ & $0.38^{\mathrm{a}}$ & $0.34^{\mathrm{a}}$ & $0.09^{\mathrm{a}}$ \\
Super & $7.28 \pm$ & $25.42 \pm$ & $14.26 \pm$ & $2.58 \pm$ & $6.62 \pm$ & $1.22 \pm$ & $0.88 \pm$ \\
Premium & $2.14^{\mathrm{a}}$ & $1.65^{\mathrm{b}}$ & $1.64^{\mathrm{a}}$ & $0.83^{\mathrm{b}}$ & $0.69^{\mathrm{a}}$ & $0.63^{\mathrm{a}}$ & $0.19^{\mathrm{a}}$ \\
\hline
\end{tabular}

Todos los valores se expresan como proporción (\%) sobre el contenido total y se presentan como media \pm desviación estándar. Letras diferentes dentro de columnas indican diferencia estadística $(p<0.05)$

Cuadro 2. Fenoles totales y capacidad antioxidante total de concentrados para perros por segmento comercial

\begin{tabular}{lcc}
\hline Segmento & $\begin{array}{c}\text { Fenoles } \\
(\mathrm{mg} \text { de ácido gálico/100 g muestra) }\end{array}$ & $\begin{array}{c}\text { ABTS } \\
(\mu \mathrm{mol} \text { Trolox/l muestra })\end{array}$ \\
\hline Popular & $160.11 \pm 49.88$ & $3146.03 \pm 564.56$ \\
Económico & $146.76 \pm 41.56$ & $2895.45 \pm 581.02$ \\
Premium & $131.69 \pm 21.59$ & $2837.60 \pm 241.72$ \\
Super Premium & $144.98 \pm 34.17$ & $2875.79 \pm 325.86$ \\
\hline
\end{tabular}

Los resultados se presentan como media \pm desviación estándar. No se observaron diferencias estadísticas $(p>0.05)$

el BHA y el EQ son ampliamente utilizados, especialmente para prevenir la oxidación de grasas y aceites (Silva y Lidon, 2016). Otro grupo de antioxidantes presentes en los alimentos son los polifenoles, una amplia variedad de moléculas biológicas que se encuentran en varias especies de plantas, los cuales desempeñan un papel importante para el normal crecimiento y desarrollo de los organismos y para su defensa contra infecciones y lesiones (Predescu et al., 2016). Las propiedades benéficas de los polifenoles están asociadas a su estructura química, la cual es capaz de interactuar con las especies reactivas de oxígeno y nitrógeno mediante mecanismos antioxidantes de transferencia de electrones (SET) y de transferencia de un átomo de hidrógeno (HAT) (Zapata et al., 2014). Adicionalmente se ha reportado que presentan actividad quelante de algunos metales (Papuc et al., 2007).

En este estudio se evaluó la actividad antioxidante de los alimentos concentrados en función de su capacidad para la neutralización de especies reactivas, sin estimar la naturaleza de los antioxidantes presentes y sus posibles implicaciones en la salud animal. 
250

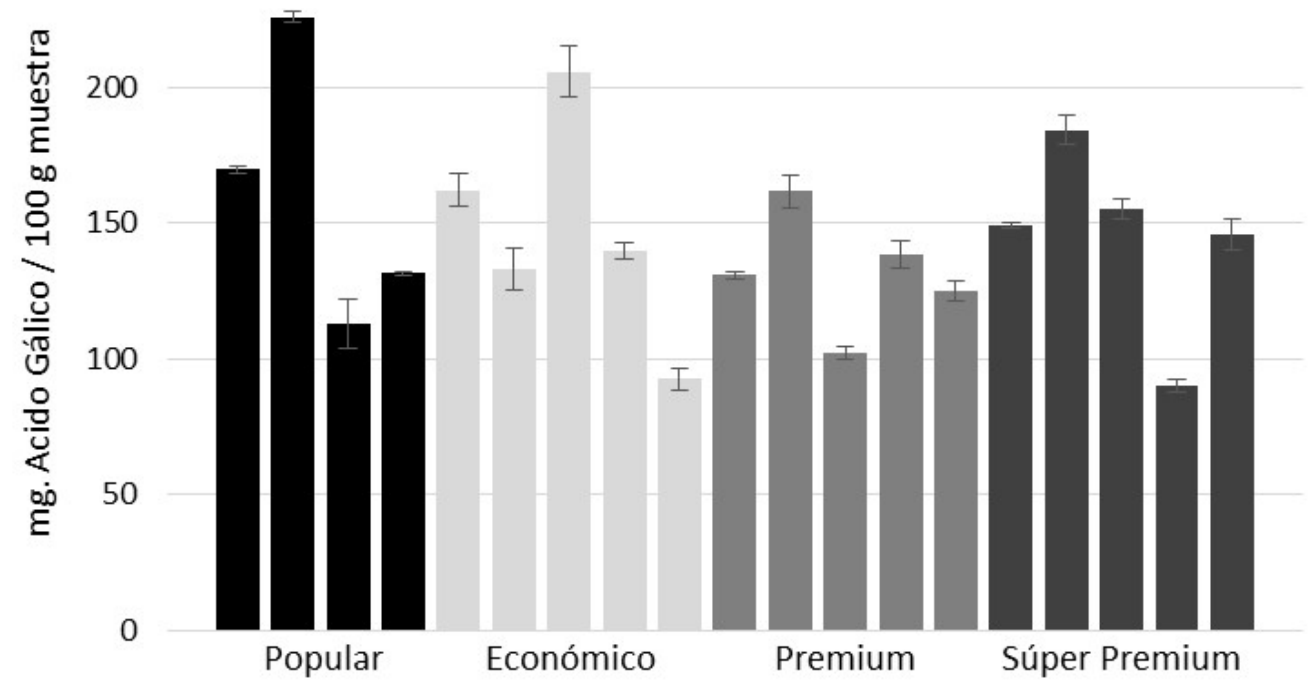

Figura 1. Contenido de fenoles totales (FT) de alimentos concentrados para perros de cuatro segmentos comerciales de Colombia

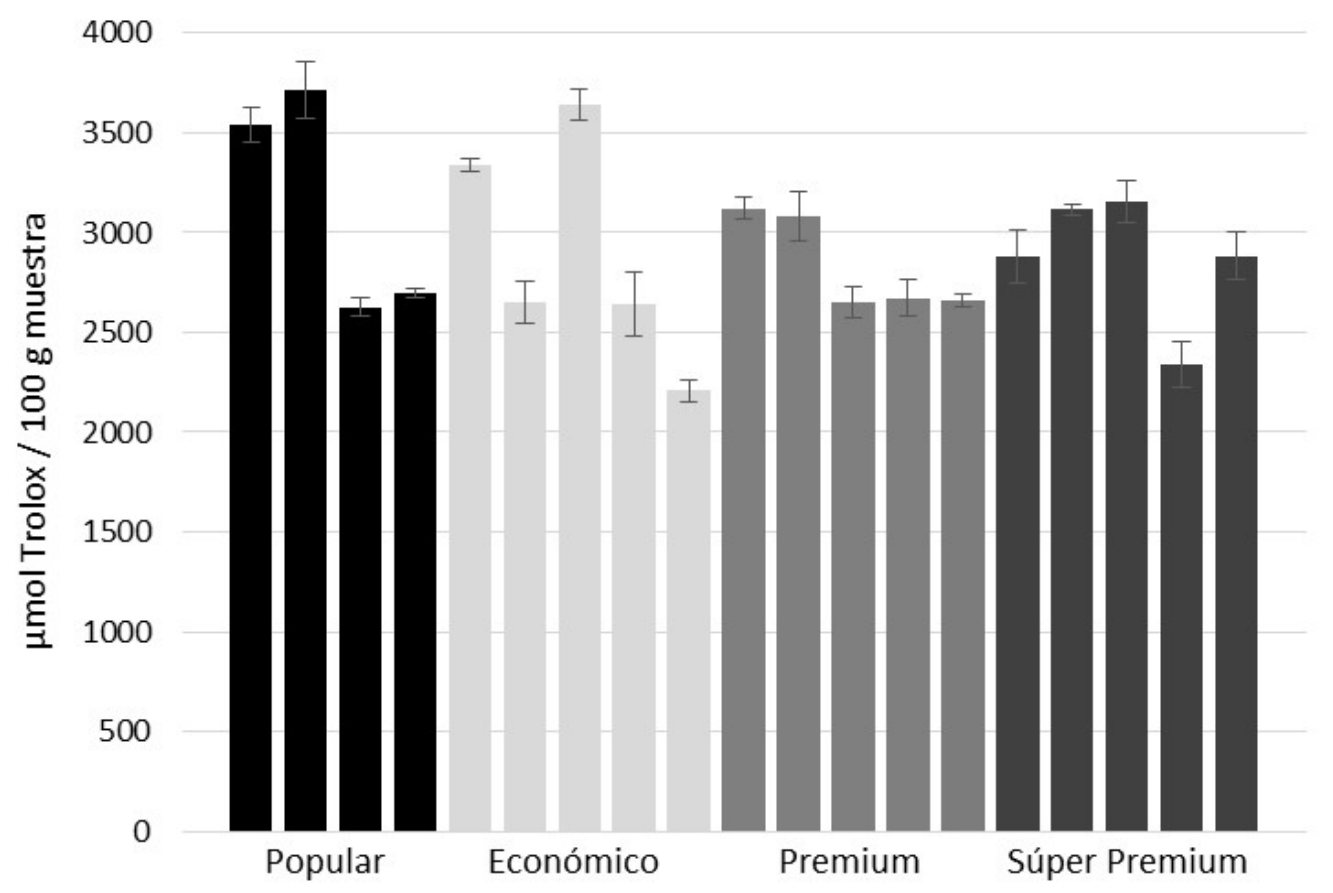

Figura 2. Capacidad antioxidante total (CAT) por ABTS de alimentos concentrados para perros de cuatro segmentos comerciales de Colombia 
No se encontraron diferencias significativas para FT y CAT entre los segmentos comerciales de alimentos concentrados para perros. Sin embargo, se observaron valores promedio más altos para FT y ABTS en los concentrados del segmento popular (Cuadro 2). Esto podría explicarse por las amplias variaciones en el aporte de fenoles y CAT entre los alimentos catalogados, incluso en un mismo segmento comercial, tal como se observa en las figuras 1 y 2 .

Adicionalmente, se encontró una correlación positiva entre el contenido de fibra cruda y los fenoles totales $(r=0.47, p<0.05)$. Se ha reportado que la fibra que se encuentra comúnmente en los alimentos para mascotas puede modificar la microflora intestinal al promover el crecimiento de bacterias comensales (Tungland, 2003; Godoy et al., 2009), disminuir el vaciamiento gástrico, las concentraciones de colesterol en sangre (Brennan y Cleary, 2005), así como el tiempo de tránsito gástrico. También puede diluir la densidad de calorías de la dieta y aumentar la saciedad (Rebello et al., 2013), la tasa de absorción de glucosa (Jenkins et al., 2008) y la excreción fecal (Wenk, 2001). No se encontraron coeficientes de correlación significativos entre otros componentes de los alimentos con FT, y en ningún caso con la CAT evaluada por la metodología ABTS.

\section{Conclusiones}

- La segmentación comercial de los concentrados para perros no está relacionada con su aporte antioxidante, valorado según la capacidad antioxidante total y el contenido total de fenoles.

- El contenido total de fenoles está asociado positivamente con el contenido de fibra cruda del concentrado, el cual se encuentra en mayor proporción en concentrados de los segmentos popular y económico.

\section{Agradecimientos}

Al personal de los laboratorios de Ciencia de los Alimentos de la Universidad Nacional de Colombia, sede Medellín, por su ayuda en la fase experimental de este estudio.

\section{Literatura Citada}

1. [AOAC] Official Methods of Analysis of AOAC International. 2019. $21^{\text {st }}$ ed. [Internet]. [Disponible en: https://www.aoac.org/aoac_prod_imis/AOAC/Publications/Offi-cial_Methods_of_Analysis/ AOAC Member/Pubs/OMA/AOAC Official_Methods_of_Analysis. aspx?hkey $=5142 \mathrm{c} 478$-ab50-4856-8939a7a491756f48

2. Arts M, Dallinga JS, Voss H, Haenen G, Bast A. 2004. A new approach to assess the total antioxidant capacity using the TEAC assay. Food Chem 88: 567570. doi: 10.1016/j.foodchem.2004.02.008

3. Baskin C, Hinchcliff K, Di Silvestro $R$, Reinhart G, Hayek M, Chew B, Burr J, et al. 2000. Effects of dietary antioxidant supplementation on oxidative damage and resistance to oxidative damage during prolonged exercise in sled dogs. Am J Vet Res 61: 886-891. doi: https://doi.org/10.2460/ajvr.2000.61.886

4. Ваuer JE. 2007. Responses of dogs to dietary omega-3 fatty acids. J Am Vet Med Assoc 231: 1657-1661. doi: 10.2460/ javma.231.11.1657

5. Blaszczyk A, Augustyniak A, Skolimowski J. 2013. Ethoxyquin: an antioxidant used in animal feed. Int $\mathbf{J}$ Food Sci 2013: 585931. doi: 10.1155/ 2013/585931

6. Brennan CS, Cleary LJ. 2005. The potential use of cereal $(1 \rightarrow 3,1 \rightarrow 4)$ - $\beta$ d-glucans as functional food ingredients. J Cereal Sci 42: 1-13. doi: 10.1016/ j.jcs.2005.01.002 
7. Corsato A, Aldrich C. 2018. The effect of sorghum fractions on apparent total tract digestibility and antioxidant capacity by dogs. Plos One 13: e0206090. doi: 10.1371/journal.pone.0206090

8. Glodde F, Günal M, Kinsel ME, AbuGhazaleh A. 2018. Effects of natural antioxidants on the stability of omega-3 fatty acids in dog food. J Vet Res 62: 103-108. doi: 10.1515/jvetres-20180014

9. Godoy MR, Bauer LL, Parsons CM, Fahey GC. 2009. Select corn coproducts from the ethanol industry and their potential as ingredients in pet foods. J Anim Sci 87: 189-199. doi: 10.2527/ jas.2007-0596

10. Hilton J. 1989. Antioxidants: function, types and necessity of inclusion in pet foods. Can Vet J 30: 682-684.

11. [ICONTEC] Instituto Colombiano de Normas Técnicas y Certificación. Norma Tecnología de Alimentos. [Internet]. Disponible en: https:// www.icontec.org/Documentos\%20compartidos/normas\%20por\%20sectores/ 67.pdf

12. Jenkins AL, Jenkins DJA, Wolever TMS, Rogovik AL, Jovanovski E, Božikov V, Raheliae D, Vuksan V. 2008. Comparable postprandial glucose reductions with viscous fiber blend enriched biscuits in healthy subjects and patients with diabetes mellitus: acute randomized controlled clinical trial. Croat Med J 49: 772-782. doi: 10.3325/ cmj.2008.49.722

13. Macotpet A, Suksawat F, Sukon P, Pimpakdee K, Pattarapanwichien E, Tangrassameeprasert R, Boonsiri P. 2013. Oxidative stress in cancer-bearing dogs assessed by measuring serum malondialdehyde. BMC Vet Res 9: 101. doi: 10.1186/1746-6148-9-101

14. Marquis A, Packer RA, Borgens RB, Duerstock BS. 2015. Increase in oxidative stress biomarkers in dogs with ascending-descending myelomalacia following spinal cord injury. J Neurol Sci 353: 63-69. doi: 10.1016/j.jns.2015.04.003
15. Milgram N, Head E, Muggenburg B, Holowachuk D, Murphey H, Estrada J, Ikeda-Douglas C, et al. 2002. Landmark discrimination learning in the dog: effects of age, an antioxidant fortified food, and cognitive strategy. Neurosci Biobehav R 26: 679-695. doi: 10.1016/S0149-7634(02)00039-8

16. Pacheco G, Bortolin R, Chaves $P$, Moreira J, Kessler A, Trevizan L. 2018. Effects of the consumption of polyunsaturated fatty acids on the oxidative status of adult dogs. J Anim Sci 96: 4590-4598. doi: 10.1093/jas/sky313

17. Pan Y, Kennedy D, Jönsson T, Milgram N. 2018. Cognitive enhancement in old dogs from dietary supplementation with a nutrient blend containing arginine, antioxidants, B vitamins and fish oil. Brit J Nutr 119: 349358. doi: 10.1017/S0007114517003464

18. Papuc C, Diaconescu C, Nicorescu V, Crivineanu C. 2007. Antioxidant properties of aromatic plant alcoholic extracts. Rom Biotech Lett 12: 35333537.

19. Passantino A, Quartarone V, Pediliggeri M, Rizzo M, Piccione G. 2014. Possible application of oxidative stress parameters for the evaluation of animal welfare in sheltered dogs subjected to different environmental and health conditions. J Vet Behav 9: 290294. doi: 10.1016/j.jveb.2014.06.009

20. Pellegrini N, Serafini M, Colombi B, Del Rio D, Salvatore S, Bianchi M, Brighenti F. 2003. Total antioxidant capacity of plant foods, beverages and oils consumed in Italy assessed by three different in vitro assays. J Nutr 133: 2812-2819. doi: 10.1093/jn/133.9.2812

21. Pompella A, Sies H, Wacker R, Brouns F, Grune T, Biesalski H, Frank J. 2014. The use of total antioxidant capacity as surrogate marker for food quality and its effect on health is to be discouraged. Nutrition 30: 791-793. doi: 10.1016/j.nut.2013.12.002 
22. Predescu NC, Nicorescu V, Petcu C, Stefan G, Papuc C. 2016. Antioxidant activity of polyphenols extracted from Hawthorn and Dog-rose fruits on linoleic acid emulsion model system compared to BHA synthetic antioxidant. Sci Works Series C Vet Med 62: 32-35.

23. Prior R, Cao G, Martin A, Sofic E, McEwen J, O'Brien C, Lischner N, et al. 1998. Antioxidant capacity as influenced by total phenolic and anthocyanin content, maturity, and variety of vaccinium species. J Agr Food Chem 46:268-2693. doi: 10.1021/j9980145d

24. Rebello CJ, Liu AG, Greenway FL, Dhurandhar NV. 2013. Dietary strategies to increase satiety. Adv Food Res 69: 105-182. doi: 10.1016/B978-012-410540-9.00003-X

25. Silva MM, Lidon FC. 2016. Food preservatives-An overview on applications and side effects. Emir J Food Agr 28: 366 373. doi: 10.9755/ejfa.2016-04-351

26. Soffler C. 2007. Oxidative stress. Vet Clin N Am-Equine 23: 135-157. doi: 10.1016/j.cveq.2006.11.004

27. Tungland BC. 2003. Fructooligosaccharides and other fructans: structures and occurrence, production, regulatory aspects, food applications, and nutritional health significance, oligosaccharides in food and agriculture. Acs Sym Ser 11: 135-152. doi: 10.1021/bk-2003-0849.-ch011

28. Turek JJ, Watkins BA, Schoenlein IA, Allen KGD, Hayek MG, Aldrich CG. 2003. Oxidized lipid depresses canine growth, immune function and bone formation. J Nutr Biochem 4: 24-31. doi: 10.1016/S0955-2863(02)00221-8

29. Wenk C. 2001. The role of dietary fibre in the digestive physiology of the pig. Anim. Feed Sci Technol, 90: 21-33. doi: 10.1016/S0377-8401(01)00194-8

30. Yang M, Chung S, Chung C, Kim D, Song W, Koo S, Chun O. 2011. Estimation of total antioxidant capacity from diet and supplements in US adults. Br J Nutr, 106: 254-263. doi: 10.1017/ S0007114511000109

31. Zapata K, Cortés F, Rojano B. 2013. Polifenoles y actividad antioxidante del fruto de guayaba agria (Psidium araca). Inf Tecnol 24: 103-112. doi: 10.4067/ S0718-07642013000500012

32. Zapata S, Piedrahita A, Rojano B. 2014. Capacidad atrapadora de radicales oxigeno (ORAC) y fenoles totales de frutas y hortalizas de Colombia. Perspect Nutr Humana 16: 25-36. 